\title{
Detección de un mosaico de trisomía 21 en líquido amniótico
}

\author{
Detection of a mosaicism of trisomy 21 in amniotic liquid
}

María Paula Prieto ${ }^{1}$, María Ximena Arteaga ${ }^{2}$, Isabel Fernandez ${ }^{3}$, Sharon Lechtig ${ }^{4}$, Carolina Ciro ${ }^{5}$, Valentina Maldonado ${ }^{6}$, Luis Gustavo Celis ${ }^{7}$

\section{Resumen}

Se analizó un resultado con alteración cromosómica tomado de una base de datos conformada por un total de 4755 muestras de líquido amniótico extraídos mediante amniocentesis con indicación de su médico tratante, riesgo sérico y edad materna avanzada. En este reporte se presenta la detección de un mosaico de trisomía 21 en líquido amniótico, mediante la técnica de Banda $\mathrm{G}$ donde se analizaron 20 metafases. Los resultados obtenidos documentan una composición cromosómica 47, XY+21 y 46, XY con una relación 9:11 respecto a las metafases analizadas, confirmándose así el diagnostico del Síndrome de Down secundario a mosaico.

Palabras claves: Síndrome de Down, mosaicismo, cariotipo anormal, técnicas de cultivo celular, diagnóstico prenatal, asesoramiento genético.

\footnotetext{
1. Facultad de Medicina. Universidad de La Sabana, Chía, Colombia. ORCID: https://orcid.org/0000-0002-4710-9105

2. Facultad de Medicina. Universidad de La Sabana, Chía, Colombia. ORCID: https://orcid.org/0000-0001-9036-2766

3. Unidad de Genética Médica. Policlínica Metropolitana, Caracas, Venezuela. ORCID: https://orcid.org/0000-0003-4268-2639

4. Facultad de Medicina. Universidad de La Sabana, Chía, Colombia ORCID: https://orcid.org/0000-0001-6292-8742

5. Facultad de Medicina. Universidad de La Sabana, Chía, Colombia. ORCID: https://orcid.org/0000-0002-9593-2788

6. Facultad de Medicina. Universidad de La Sabana, Chía, Colombia. ORCID: https://orcid.org/0000-0001-9036-2766

7. Facultad de Medicina. Universidad de La Sabana, Chía, Colombia. ORCID: https://orcid.org/0000-0002-0338-6258.

Correspondencia: luiscelis@unisabana.edu.co
} 


\begin{abstract}
A result with chromosomal alteration was analyzed from a database consisting of a total of 4755 samples of amniotic fluid extracted by amniocentesis with indication of the attending physician, serum risk and advanced maternal age. This report presents the detection of a mosaicism of trisomy 21 in amniotic fluid, using G- Banding where 20 metaphases were analyzed. The results obtained document a chromosomal composition 47, XY +21 and 46, $\mathrm{XY}$ with a 9:11 ratio with respect to the metaphases analyzed, confirming the diagnosis of Down syndrome secondary to mosaicism.
\end{abstract}

Keywords: Down's Syndrome, Mosaicism, Abnormal Karyotype, Cell Culture Techniques, Prenatal Diagnosis, Genetic Counseling.

\title{
Introducción
}

La trisomía 21, llamada comúnmente síndrome de Down (SD), es la aneuploidía más común a nivel mundial y la principal causa de discapacidad cognitiva de origen genético (1). La prevalencia de SD difiere de un país a otro y, a veces, de un distrito a otro dentro del mismo país. Sin embargo; según la Organización Mundial de la Salud, la incidencia global predecible generalmente es de 1 a 10/1000 nacidos vivos en todo el mundo (2). En Colombia se estima que la incidencia está en 17,82 por cada 10.000 nacidos vivos, promedio un poco mas bajo del promedio de Latinoamérica el cual está en 18,8 por cada 10.000 nacidos vivos (3).

Es probable que las personas afectadas tengan una serie de afecciones médicas que afectan los sistemas cardíaco, digestivo y respiratorio. Las causas más comunes de mortalidad en el SD son las enfermedades respiratorias (neumonía), enfermedades cardíacas congénitas, trastornos circulatorios y demencia (4).

Desde una perspectiva epidemiológica, otros dos trastornos son importantes porque se ha informado un exceso tanto en los individuos afectados como en sus madres: la enfermedad de Alzheimer y la enfermedad de la tiroides (5).

La enfermedad cuenta con variantes citogenéticas que cambian las manifestaciones clínicas de la enfermedad. La trisomía 21 es la variante más común, presente en el $95 \%$ de los casos. Sin embargo, la enfermedad se puede presentar secundaria a translocación robertsoniana, mosaicismo y trisomía parcial $(6,7,8)$.

El diagnóstico prenatal y postnatal de este síndrome se realiza mediante el cariotipo, a través del cual se identifica con exactitud la 
variante citogenética, y permite realizar una adecuada asesoría genética, en especial en la evaluación del posible riesgo de aparición de nuevos casos en la familia (1). Con base en lo anterior, la amniocentesis, como parte del diagnóstico prenatal citogenético, cobra vital importancia al ser el método principal para la identificación de anomalías cromosómicas, ya sea estructurales o de número (9).

El objetivo de este trabajo es describir un caso de mosaico de trisomía 21 documentado en líquido amniótico, de una paciente con 37 ańos y 17 semanas de gestación, en quien se indicó el análisis citogenético ante edad materna avanzada.

\section{Metodología}

Se toma una base de datos conformada por un total de 4755 líquidos amnióticos obtenidos mediante amniocentesis con indicación de su médico tratante y previo consentimiento informado, riesgo sérico y edad materna avanzada, no presentando antecedentes familiares de enfermedades genéticas ni consanguinidad. Las muestras de líquido amniótico seleccionada (líquido claro y punción única), se le procedió a centrifugar a $800 \mathrm{rpm}$, descartando el sobrenadante y posteriormente las células se resuspendieron hasta 3 mililitros en medio de cultivo celular (Chang Amnio, Irvine Scientific) y colocado en incubadora de $\mathrm{CO} 2(5 \%)$ y temperatura de $37{ }^{\circ} \mathrm{C}$ durante 7 días, posteriormente a esta células se les procedió a realizar el Bandeo de las láminas mediante la técnica de Banda $G$ y se analizaron 20 metafases.

\section{Resultados}

Al analizar los 4755 líquidos amnióticos, se identificaron 263 casos que presentaban anomalías cromosómicas, encontrándose 40 anomalías de tipo estructural (deleciones, inversiones, translocaciones y duplicaciones), y 211 anomalías del tipo numéricas (aneuploidías y poliploidías). Una de las muestras presentó una composición cromosómica 47, XY+21 y 46, XY con una relación 9:11 de las metafases analizadas, el cual corresponde a un mosaico de trisomía 21, la cual culminó en la perdida de la gestación durante el segundo trimestre (ver Figura $1 b)$. 
Figura 1. Cariotipo de células extraídas de muestra de líquido amniótico.

Composición cromosómica 46, XY (a) y 47, XY+21 (b). Mosaico de trisomía 21.

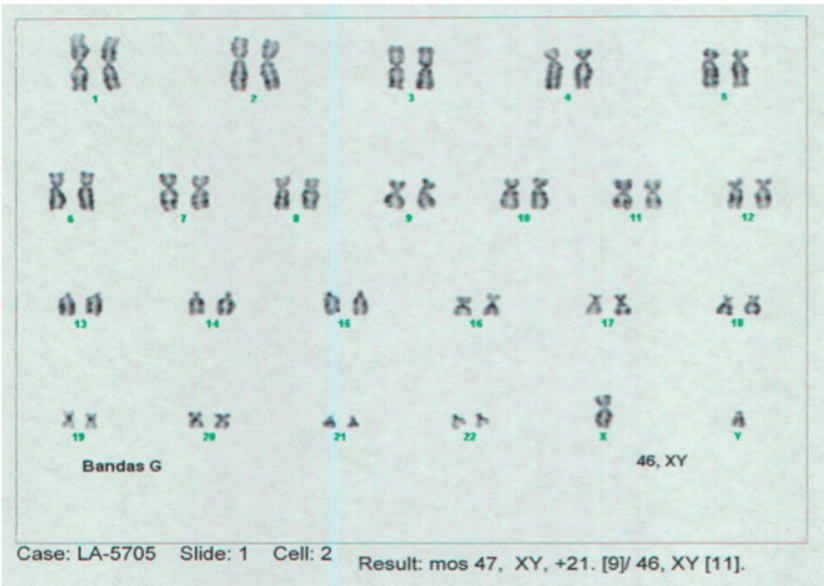

(a)

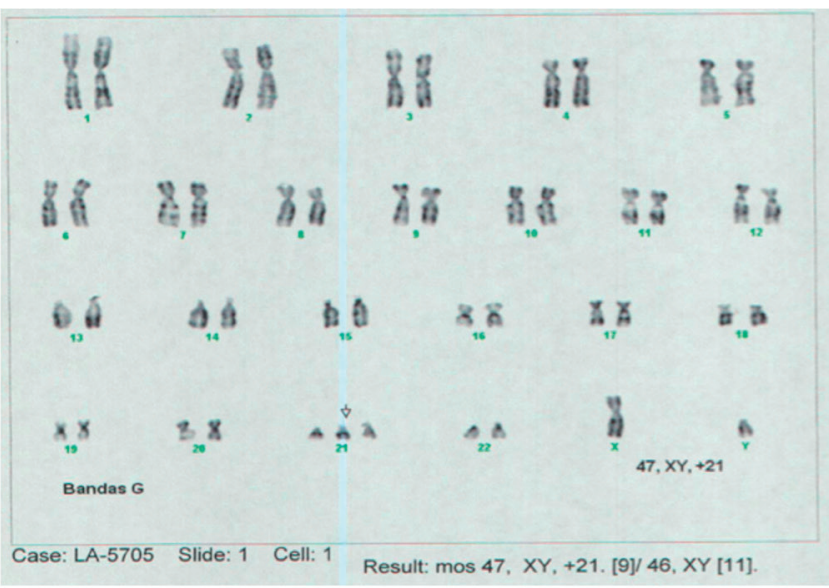

(b)

\section{Discusión}

Existen las siguientes variantes citogenéticas causantes del SD: la trisomía 21, que constituye el 90-95\% de los casos, con cariotipo $47, \mathrm{XX}$ o $\mathrm{XY}+21$; las translocaciones robertsonianas, presentes en el $4 \%$ de los casos donde se da un re-arreglo de un cromosoma 21 con otro de los cromosomas acrocéntricos (de los grupos D o G), con un cariotipo $46, \mathrm{XX}$ o $\mathrm{XY}, \operatorname{rob}(\mathrm{D}$ o $\mathrm{G} ; 21)$ $(q 10 ; q 10),+21 \quad(6,7)$. Finalmente, existe una variante menos común dada por mosaicismo, presente en aproximadamente el 1\% de los casos, donde existen dos o más líneas celulares diferentes en el mismo individuo. Finalmente se puede encontrar la trisomía parcial, presente en menos del $1 \%$ de los casos, se genera en la región $21 \mathrm{q} 22.3$, con cariotipo 46,XX o XY,dup(21)(q22.3) (8).

El caso presentado presenta un paciente con SD causado por una variante citoge- nética poco habitual. El mosaico 21 es una forma de presentación que corresponde aproximadamente al $1 \%$ de los casos con esta enfermedad. Se estima que la incidencia de mosaicismo en síndrome de Down va desde 1 en 16.670 hasta 1 en 41.670 nacidos vivos (6).

Se han descrito múltiples factores de riesgo para el SD. El principal factor de riesgo es la edad materna avanzada. La prevalencia de los nacimientos aumenta rápidamente con la edad, particularmente después de los 30 años. En consecuencia, la edad materna promedio en los nacimientos con SD es aproximadamente 5 años mayor que los nacimientos no afectados (5). Sin embargo, en un estudio realizado en 29.000 pacientes, de los cuales 322 casos eran mosaicismos, se evidenció que la distribución de la edad materna para los casos de trisomía 21 con 
mosaicismo difiere de la simple trisomía 21, con madres mucho más jóvenes (10). Alrededor de dos tercios de los diagnósticos de trisomía 21 con mosaicismo parecen tener los mismos riesgos asociados con la edad que la trisomía 21 simple, mientras que un tercio parece ser independiente de la edad materna. Esto indica que hay otro factor involucrado en la presencia de mosaicismo no asociado con la edad materna (10). En el caso presentado, al evidenciar que ninguno de los padres presentó aneuploidías, se cree que el factor de riesgo más probable fue la edad materna avanzada. No obstante, se necesitan más estudios para determinar factores de riesgo asociados a la aparición de esta variante citogenética en particular.

El aborto es la complicación más frecuente en la gestación, cerca del 10-15\% de los embarazos clínicamente reconocidos terminan en abortos espontáneos y aproximadamente el $50 \%$ de estas pérdidas son secundarias a anomalías genéticas (tanto en número como estructura) $(11,12,13)$. Este evento genético puede enfocarse a través de dos puntos de vista: anomalías genéticas de los progenitores y anomalías genéticas fetales. La primera contribuye a un mayor riesgo de aborto espontáneo en embarazos sucesivos incluyendo alteraciones en el cariotipo, condiciones de carácter dominante o recesivo, así como mutaciones en genes responsables de la coagulación o el metabolismo de folatos; y en la segunda, encontramos condiciones de novo y mutaciones heredadas. Se estima que el $50 \%$ de los abortos espontáneos en el primer trimestre son por cromosomopatías de novo $(14,15)$. La mitad de los abortos espontáneos tempranos $(<12$ semanas de edad gestacional) son causados por anomalías cromosómicas fetales, y casi un tercio en los abortos espontáneos del segundo trimestre como se informó en una revisión anterior (16). Los estudios citogenéticos han demostrado que la mayoría de estas anomalías son anomalías cromosómicas numéricas (86\%), y una minoría de los casos es causada por anomalías cromosómicas estructurales $(6 \%)$ y mosaicismo cromosómico (8\%) (16). Sin embargo, no hay estudios que demuestren una relación directa entre el aborto temprano y la presencia de un mosaico de trisomía 21.

Si bien el SD secundario a mosaicismo no es el más frecuente, se ha demostrado que es subdiagnosticado, debido a que, aparte del reto para el diagnóstico clínico, existen desafíos para su diagnóstico a nivel de técnicas citogenéticas. En caso de un mosaicismo con una baja proporción de la línea celular alterada, las manifestaciones fenotípicas son sutiles y conducen a falla en el reconocimiento mediante el examen físico. En la literatura se estima que solo el 37,5\% de estos pacientes con esta variante citogenética se detectan por las manifestaciones clínicas (17). Adicionalmente, aparte del reto para el diagnóstico clínico a nivel de técnicas diagnósticas citogenéticas lo es también. Al tomar muestras de un solo tejido y una cantidad tan pequeńa, el sesgo de no detección aumenta, sumado a que el 
número de células evaluadas es insuficiente para detectar las líneas celulares comprometidas si la relación es baja $(6,17,18)$.

El síndrome de Down, siendo la aneuploidía más frecuente en el ser humano, es la principal causa de discapacidad cognitiva de origen genético (1); los pacientes con esta condición tienen un fenotipo caracterizado por talla baja, obesidad, pliegue palmar único, hipotonía, entre otros (19). Sin embargo, debido a la variabilidad citogenética causante de esta enfermedad, la presentación clínica es diversa y depende de la presencia de múltiples factores genéticos en especial en el caso de los mosaicismos (20). Las manifestaciones clínicas del SD debido a mosaicismo son muy diversas, desde una presentación fenotípica comparable a la de los individuos que tienen trisomía 21 sin mosaico hasta un fenotipo que pasaría desapercibido y no sugestivo de alguna alteración (6). En los casos que se presentan manifestaciones fenotípicas sutiles, puede ocurrir una falla en el reconocimiento mediante el examen físico (17). Adicionalmente, se cree que estas diferencias fenotípicas reflejan principalmente un número variable de células trisómicas en diferentes personas, así como la variación de tejido a tejido dentro de una persona (6).

En este contexto, el diagnóstico prenatal es una herramienta comúnmente utilizada para identificar anomalías cromosómicas, a través de la aplicación de pruebas diagnósticas invasivas y no invasivas que permiten determinar tempranamente altera- ciones genéticas. Dentro de las anteriores, la amniocentesis con el subsecuente cultivo celular y realización de cariotipo es la técnica que más se realiza dado su bajo índice de riesgo, tanto para la madre como para el feto, por encima de otros procedimientos como la biopsia de vellosidades coriales $(21,22,23)$. Sin embargo, no es una prueba exenta de riesgos como la pérdida de la gestación, el trabajo de parto pretérmino y la ruptura prematura de membranas, entre otros $(22,24)$.

Cuando se identifica una alteración cromosómica en líquido amniótico, es necesario brindar asesoría genética estricta por el médico genetista a los progenitores. Esta actividad debe incluir necesariamente información a los padres sobre el diagnóstico, el pronóstico del producto, las opciones de seguimiento o terminación del embarazo, también allí se abordan las medidas que deberán tomarse al momento del nacimiento, así como la evaluación del riesgo de recurrencia de enfermedades hereditarias $(9,22)$. La literatura recomienda que en estos casos si la gestación llega a término, el cariotipo debe repetirse al nacimiento sobre todo en aquellos casos en que el estudio quedó inconcluso, o para corroborar el origen de la alteración y poder brindar una asesoría genética adecuada (9). 


\section{Referencias}

1. Vergara Estupiñán E. J. , Forero-Castro R. M, Moreno Granados J. I. Estudio descriptivo-transversal del síndrome de Down en pacientes de Boyacá (Colombia). Revista Ciencia en Desarrollo. 2014; 5 (2): 187-195.

2. Mohammed Al-Biltagi. Down syndrome from Epidemiologic Point of View. EC Paediatrics. 2015; 2: 82-91.

3. Nazher J, Cifuentes L. Malformaciones congénitas en Chile y Latino América: Una visión epidemiológica del ECLAMC del período 1995-2008. Rev Med Chile. 2011; 139(1): 72-78.

4. Arumugam A, Raja K, Venugopalan M, Chandrasekaran B, Kovanur Sampath K, Muthusamy H et al. Down syndrome-A narrative review with a focus on anatomical features. Clin Anat. 2016; 29(5): 56877.

5. Cuckle H, Arbuzova S. Epidemiology and Genetics of Human Aneuploidy. En: Leung P, Human Q. Reproductive and Prenatal Genetics. $1^{\mathrm{a}} \mathrm{ed}$. Reino Unido: American Press; 2019. p. 529-551.

6. Papavassiliou P, Charalsawadi C, Rafferty K, Jackson-Cook C. Mosaicism for trisomy 21: A review. Am J Med Genet A. 2015; 167A(1): 26-39.

7. Frías S, Ramos S, Molina B, del Castillo V, Mayén DG. Detection of mosaicism in lymphocytes of parents of free trisomy 21 offspring. Mutation Research. 2002; 520 (1): 25-37.

8. Hultén MA, Patel SD, Tankimanova M, Westgren M, Papadogiannakis N, Jonsson AM. On the origin of trisomy 21 Down syndrome. Molecular Cytogenetics. 2008; 1: 21.

9. Giraldo A, Arias A, León MC, Fernández I, Celis LG. Triploidía (69 xxx): reporte de caso. Revista de Medicina e Investigación UAEMéx. 2018; 6 (1): 2327.

10. Morris JK. Trisomy 21 mosaicism and maternal age. Am J Med Genet A. 2012; 158A (10): 2482-4.
11. Dória S, Carvahlo F, Ramalho C, et al. An efficient protocol for the detection of chromosomal abnormalities in spontaneous miscarriages or fetal deaths. Eur J Obstet Gynecol Reprod Biol. 2009; 147: 144-150.

12. Chan-Wei J, Li W, Yong-Lian L, Rui S, Li-Yin Z, Lan $\mathrm{Y}$ et al. Aneuploidy in Early Miscarriage and its Related Factors. Chinese Medical Journal. 2015; 128 (20): 2272-2776.

13. Warburton D. Cytogenetics of reproductive wastage: from conception to birth. Medical Cytogenetics. 2000; 213-246.

14. Simpson JL. Causes of fetal wastage. Clin Obstet Gynecol. 2007; 50: 10-30.

15. Kacprzak M, Chrzanowska M, Skoczylas B, Moczulska H, Borowiec M, Sieroszewski P. Genetic causes of recurrent miscarriages. Ginekologia Polska. 2016; 87(1): 722-726.

16. Van den Berg MM, Van Maarle MC, Van Wely M, Goddijn M. Genetics of early miscarriage. Biochim Biophys Acta. 2012; 1822(12): 1951-9.

17. Devlin L, Morrison PJ. Accuracy of the clinical diagnosis of Down syndrome. Ulster Med J. 2004; 73: 4-12.

18. Chen CP, Wang YL, Chern SR, Wu PS, Chen YN, Chen SW et al. Prenatal diagnosis and molecular cytogenetic characterization of low-level true mosaicism for trisomy 21 using uncultured amniocytes. Taiwan J Obstet Gynecol. 2016; 55(2): 285-7.

19. Asim A, Kumar A, Muthuswamy S, Jain S, Agarwal S. Down syndrome: an insight of the disease. Journal of Biomedical Science. 2015; 22: 41- 50.

20. Díaz-Cuéllar S, Yokoyama-Rebollar E, Del Castillo-Ruiz V. Genómica del síndrome de Down. Acta pediátrica de México. 2016; 37(5): 289-296.

21. Krstic N, Obic ${ }^{\vee} a n$ S. Current landscape of prenatal genetic screening and testing. Birth Defects Res. 2019;1-11. 
22. Gómez-Puente V, Esmer-Sánchez M, Quezada-Espinoza C, Martínez-de Villarreal C. Estudio citogenético en líquido amniótico, Experiencia de 7 años en la Facultad de Medicina y Hospital Universitario, UANL. Medicina Universitaria. 2012; 14: 23-29.

23. Torres, C., Usta, C., Mancilla, L., Fernández, I., \& Celis, L. (2018). Diagnóstico prenatal de inversión pericentrica del cromosoma cinco de novo en una paciente con gestación a término sin complicaciones posteriores. NOVA, 16(30), 75-80. https://doi. org/10.22490/24629448.2837

24. Chang HP, Chion JY, Chen JY, Su PH. Prenatal cytogenetic diagnosis in Taiwan: a nationwide population-based study. J Matern Fetal Neonatal Med. 2017; 30(21): 2521-2528. 